Research Article

\title{
A Comparison of Normal Cone Conditions for Homotopy Methods for Solving Inequality Constrained Nonlinear Programming Problems
}

\author{
Zhengyong Zhou $(\mathbb{D}$ ) and Ting Zhang \\ School of Mathematics and Computer Sciences, Shanxi Normal University, Shanxi 041004, China \\ Correspondence should be addressed to Zhengyong Zhou; zzy198300@163.com
}

Received 30 January 2020; Revised 16 June 2020; Accepted 17 June 2020; Published 4 July 2020

Academic Editor: Eugen Radu

Copyright (c) 2020 Zhengyong Zhou and Ting Zhang. This is an open access article distributed under the Creative Commons Attribution License, which permits unrestricted use, distribution, and reproduction in any medium, provided the original work is properly cited.

\begin{abstract}
Homotopy methods are powerful tools for solving nonlinear programming. Their global convergence can be generally established under conditions of the nonemptiness and boundness of the interior of the feasible set, the Positive Linear Independent Constraint Qualification (PLICQ), which is equivalent to the Mangasarian-Fromovitz Constraint Qualification (MFCQ), and the normal cone condition. This paper provides a comparison of the existing normal cone conditions used in homotopy methods for solving inequality constrained nonlinear programming.
\end{abstract}

\section{Introduction}

In this paper, we consider the following inequality constrained nonlinear programming (ICNLP) problem:

$$
\begin{array}{cl}
\min & f(x), \\
\text { s.t. } & g_{i}(x) \leq 0, i=1, \cdots, m,
\end{array}
$$

where $x \in R^{n}$ is the variable, $f: R^{n} \longrightarrow R$ and $g_{i}: R^{n} \longrightarrow R$, $i=1, \cdots, m$, are three times continuously differentiable.

Over the past few decades, the theory, algorithms, and applications of nonlinear programming have been rapidly developed, and many numerical methods have been proposed, such as augmented Lagrangian methods, sequential quadratic programming methods, reduced gradient methods, interior point methods, and homotopy methods. Homotopy methods are powerful numerical methods for solving many nonlinear problems. The primary advantage of homotopy methods is that their global convergence can be established under fairly weak assumptions, and the starting points can be chosen rather freely. A comprehensive introduction of the homotopy methods can be found in, e.g., the books $[1,2]$. The first homotopy method for solving nonlinear programming was proposed for general convex programming in [3]. Among these available homotopy methods for solving nonlinear programming, most of them are designed for solving nonconvex ICNLP problems.

In 1995, the combined homotopy interior point (CHIP) method was proposed for solving nonconvex ICNLP problems in $[4,5]$. Hereafter, many modified CHIP methods have been proposed for nonconvex ICNLP problems. The global convergence of these homotopy methods can be generally established under three conditions on the original problem: the nonemptiness and boundedness of the interior of the feasible set; the Positive Linear Independent Constraint Qualification (PLICQ), which is equivalent to the Mangasarian-Fromovitz Constraint Qualification (MFCQ) (see [6]); and one type of normal cone conditions, which guarantees the boundedness of the homotopy path near the starting hyperplane. It is well known that the first two conditions are generally used in numerical methods for solving nonlinear programming. The normal cone conditions are generalization of the convexity of the feasible set and extend these homotopy methods from convex programming to nonconvex programming. In addition, a probability-one 
homotopy method was also proposed for solving nonconvex ICNLP problems in [7]; its global convergence was established under the nonemptiness and boundedness of a parametrized feasible set, Arrow-Hurwicz-Uzawa constraint qualification, and that the homotopy path does not go to infinity near the starting hyperplane. In recent years, these homotopy methods have been extended to fixed point problems, variational inequalities, semidefinite programming, multiobjective programming, constrained sequential minimax problems, and so on.

In this paper, we present the typical normal cone conditions for homotopy methods for solving ICNLP problems, along with the corresponding homotopy maps and global convergence. We give a comparison of four normal cone conditions, including the normal cone condition, the quasinormal cone condition, the pseudocone condition, and the weak normal cone condition. Their relations are discussed in detail for the first time. Some typical nonconvex sets are presented. The comparison can help us to identify features of these normal cone conditions and the corresponding homotopy methods and may motivate us to give some improved homotopy methods for specialized nonconvex programming.

To obtain our results, we conclude this section with some notations. Throughout this paper, $\Omega=\left\{x \in R^{n} \mid g_{i}(x) \leq 0\right.$, $i=1, \cdots, m\}$ represents the feasible set of the ICNLP problem (1); $\Omega^{0}=\left\{x \in R^{n} \mid g_{i}(x)<0, i=1, \cdots, m\right\}$ denotes the interior of $\Omega$; $\partial \Omega=\Omega \Omega^{0}$ means the boundary of $\Omega$. $I(x)=\left\{i \in\{1, \cdots, m\} \mid g_{i}(x)=0\right\} \quad$ represents the active index set of inequality constraints at $x . R_{+}^{m}$ and $R_{++}^{m}$ denote the nonnegative and positive quadrant of $R^{m}$, respectively. $I$ indicates the identity matrix. $\|\cdot\|$ denotes the Euclidean norm. For a function $F: R^{n} \longrightarrow R^{m}, F^{-1}(C)=$ $\left\{x \in R^{n} \mid F(x) \in C\right\}$ is the inverse of the set $C \in R^{m}$; the $n \times m$ matrix $\nabla F(x)$, whose $(i, j)$ th element is $\partial F_{j}(x) / \partial x_{i}$, is the transpose of the Jacobian of $F$.

Assumption 1. $\Omega^{0}$ is nonempty and bounded.

Assumption 2. The Positive Linear Independent Constraint Qualification holds: for any $x \in \partial \Omega, y_{i} \geq 0, i \in I(x)$,

$$
\sum_{i \in I(x)} y_{i} \nabla g_{i}(x)=0, y_{i} \geq 0, i \in I(x), \quad \text { imply } y_{i}=0, i \in I(x)
$$

\section{Normal Cone Conditions and Homotopy Methods for ICNLP Problems}

There exist four typical normal cone conditions in homotopy methods for solving ICNLP problems in literatures; related results will be introduced in this section.

For the nonconvex ICNLP problem (1), the first homotopy method, called the combined homotopy interior point (CHIP) method, was proposed in $[4,5]$. The combined homotopy map is constructed as

$$
H(x, y, t)=\left(\begin{array}{c}
(1-t)(\nabla f(x)+\nabla g(x) y)+t\left(x-x^{0}\right) \\
Y g(x)-t Y^{0} g\left(x^{0}\right)
\end{array}\right)
$$

where $(x, y, t) \in \Omega \times R_{+}^{m} \times[0,1], \quad\left(x^{0}, y^{0}\right) \in \Omega^{0} \times R_{++}^{m}, \quad Y$ and $Y^{0}$ are diagonal matrices with the $i$ th diagonal elements $y_{i}$ and $y_{i}^{0}$ for $i=1, \cdots, m$, respectively. Under Assumption 1, the assumption that $\left\{\nabla g_{i}(x) \mid i \in I(x)\right\}$ has a full column rank for any $x \in \partial \Omega$, which can be replaced by Assumption 2 , and the assumption that $\Omega$ satisfies the normal cone condition (see Definition 3), for almost all $\left(x^{0}, y^{0}\right) \in \Omega^{0} \times R_{++}^{m}$, the zero point set of (3) defines a smooth homotopy path $\Gamma_{\left(x^{0}, y^{0}\right)} \subset \Omega^{0} \times R_{++}^{m} \times(0,1]$, which starts from $\left(x^{0}, y^{0}, 1\right)$ and approaches to the hyperplane $t=0$. For any limit point $\left(x^{*}\right.$, $\left.y^{*}, 0\right)$ of $\Gamma_{\left(x^{0}, y^{0}\right)}, x^{*}$ is a KKT point of the problem (1), $y^{*}$ is the corresponding Lagrange multiplier.

Definition 3. (normal cone condition (NCC), see $[4,5]$ ). For any $x \in \partial \Omega$, if

$$
\left\{x+\sum_{i \in I(x)} y_{i} \nabla g_{i}(x) \mid y_{i} \geq 0, i \in I(x)\right\} \bigcap \Omega^{0}=\varnothing
$$

then, $\Omega$ is said to satisfy the NCC.

According to Definition 3, the NCC means that, for any $x \in \partial \Omega$, the set $\left\{x^{0}-x \mid x^{0} \in \Omega^{0}\right\}$ does not intersect with the cone $\left\{\sum_{i \in I(x)} y_{i} \nabla g_{i}(x) \mid y_{i} \geq 0, i \in I(x)\right\}$. Moreover, if $g_{i}: R^{n}$ $\longrightarrow R, i=1, \cdots, m$, are convex, then $\Omega$ satisfies the normal cone condition; however, the reciprocal implication is not true, a typical counterexample is $\Omega=\left\{x \in R^{2} \mid-\left(x_{1}-4\right)^{2}-\right.$ $\left.x_{2}^{2}+8 \leq 0,-2 x_{1}+x_{2}^{2} \leq 0, x_{1}-3 \leq 0\right\}$ (see Figure 1). In [8], a modified CHIP method was presented. The homotopy map is defined as

$$
H(x, y, t)=\left(\begin{array}{c}
(1-t)\left(\nabla f(x)+\nabla g(x) y+t \eta(x) y^{2}\right)+t\left(x-x^{0}\right) \\
Y g(x)-t Y^{0} g\left(x^{0}\right)
\end{array}\right)
$$

where $(x, y, t) \in \Omega \times R_{+}^{m} \times[0,1],\left(x^{0}, y^{0}\right) \in \Omega^{0} \times R_{++}^{m}$, and $\eta(x)$ is a positive independent map with respect to $\nabla g(x)$ (see Definition 4). Under Assumption 1, the assumption that $\left\{\nabla g_{i}(x) \mid i \in I(x)\right\}$ are linear independent for any $x \in \partial \Omega$, which can be also replaced by Assumption 2, and the assumption that $\Omega$ satisfies the quasinormal cone condition related to the positive independent map $\eta(x)$ (see Definition 5), for almost all $\left(x^{0}, y^{0}\right) \in \Omega^{0} \times R_{++}^{m}$, the global convergence of a smooth homotopy path $\Gamma_{\left(x^{0}, y^{0}\right)} \subset \Omega^{0} \times$ $R_{++}^{m} \times(0,1]$ starting from $\left(x^{0}, y^{0}, 1\right)$ and approaching to the hyperplane $t=0$ can be established; then, a KKT point $x^{*}$ with the corresponding Lagrange multiplier $y^{*}$ can be obtained. 


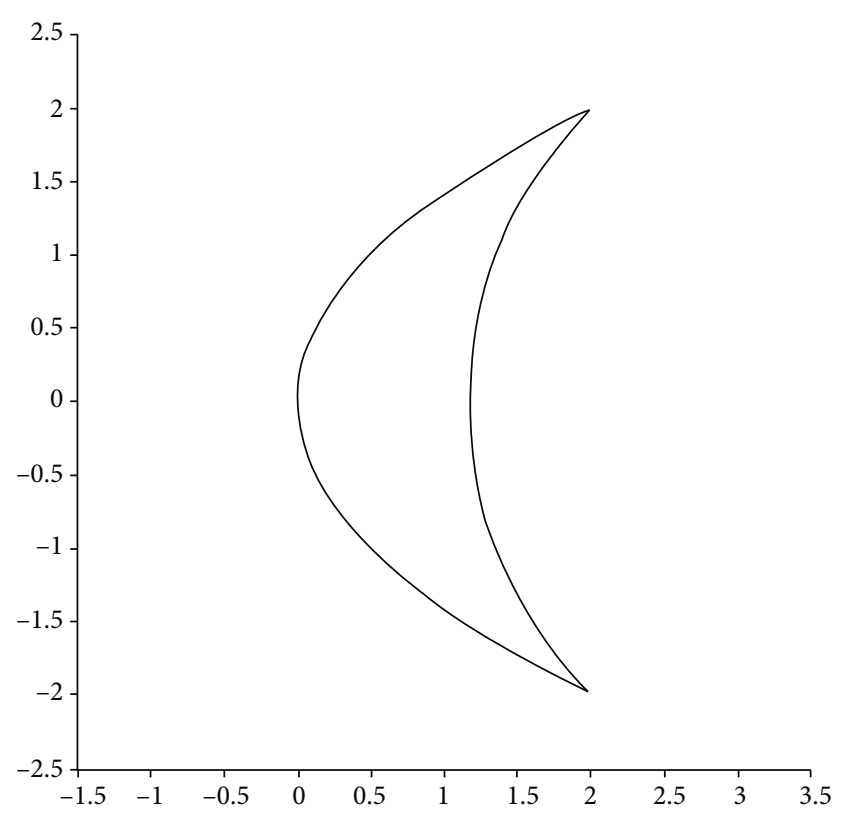

Figure 1: The set $\Omega=\left\{x \in R^{2} \mid-\left(x_{1}-4\right)^{2}-x_{2}^{2}+8 \leq 0,-2 x_{1}+x_{2}^{2}\right.$ $\left.\leq 0, x_{1}-3 \leq 0\right\}$.

Definition 4 (positive independent map, see [8]). If there exist smooth maps $\eta_{i}(x): R^{n} \longrightarrow R^{n}$ for $i=1, \cdots, m$, such that, for any $x \in \partial \Omega$,

$$
\begin{aligned}
\sum_{i \in I(x)}\left(y_{i} \nabla g_{i}(x)+\alpha_{i} \eta_{i}(x)\right) & =0, y_{i} \geq 0, \alpha_{i} \geq 0, i \in I(x), \text { imply } y_{i} \\
& =0, \alpha_{i}=0, i \in I(x)
\end{aligned}
$$

then, $\eta(x)=\left(\eta_{1}(x), \cdots, \eta_{m}(x)\right)$ is said to be a positive independent map with respect to $\nabla g(x)$.

Definition 5 (quasinormal cone condition (QNCC) [8]). If there exists a smooth positive independent map $\eta(x)=$ $\left(\eta_{1}(x), \cdots, \eta_{m}(x)\right)$ with respect to $\nabla g(x)$ such that, for any $x \in \partial \Omega$,

$$
\left\{x+\sum_{i \in I(x)} \alpha_{i} \eta_{i}(x) \mid \alpha_{i} \geq 0, i \in I(x)\right\} \bigcap \Omega^{0}=\varnothing,
$$

then, $\Omega$ is said to satisfy the QNCC related to $\eta(x)$.

According to Definition 5, the QNCC means that, for any $x \in \partial \Omega$, the set $\left\{x^{0}-x \mid x^{0} \in \Omega^{0}\right\}$ does not intersect with the cone $\left\{\sum_{i \in I(x)} \alpha_{i} \eta_{i}(x) \mid \alpha_{i} \geq 0, i \in I(x)\right\}$.

In [9], another modified CHIP method was proposed with the homotopy map

$$
H(x, y, t)=\left(\begin{array}{c}
\left.(1-t)(\nabla f(x)+\nabla g(x) y)+\sum_{i=1}^{m} \eta_{i} x,(1-t) y_{i}^{2}\right)+t\left(x-x^{0}\right) \\
Y g(x)-t Y^{0} g\left(x^{0}\right)
\end{array}\right),
$$

where $(x, y, t) \in \Omega \times R_{+}^{m} \times[0,1],\left(x^{0}, y^{0}\right) \in \Omega^{0} \times R_{++}^{m}$, and $\eta(x$, $\left.(1-t) y^{2}\right)=\left(\eta_{1}\left(x,(1-t) y_{1}^{2}\right), \cdots, \eta_{m}\left(x,(1-t) y_{m}^{2}\right)\right)$ is a consistent hair map (see Definition 7 ). Similarly to the homotopy methods in $[1,6]$, under Assumption 1, the assumption that $\left\{\nabla g_{i}(x) \mid i \in I(x)\right\}$ has a full column rank for any $x \in \partial \Omega$, and the assumption that $\Omega$ satisfies the pseudocone condition with respect to the consistent hair map $\eta(x, z)$ (see Definition 8), the global convergence can be established.

Definition 6 (hair map, see [9]). For $i=1, \cdots, m$, the map $\eta_{i}\left(x, z_{i}\right): R^{n} \times R_{+} \longrightarrow R^{n}$ is said to be a hair map of $\Omega_{i}=$ $\left\{x \in R^{n} \mid g_{i}(x) \leq 0\right\}$, if

$$
\begin{gathered}
\text { (1) } \eta_{i}(x, 0)=0 \text { and for any } x \in \partial \Omega_{i}=\partial \Omega \cap \Omega_{i} \\
\eta_{i}\left(x, z_{i}\right)=0 \Leftrightarrow z_{i}=0,
\end{gathered}
$$

(2) For any $x \in \partial \Omega_{i}, \lim _{z_{i} \rightarrow \infty}\left\|\eta_{i}\left(x, z_{i}\right)\right\|=\infty$

Definition 7 (consistent hair map, see [9]). The map $\eta(x, z)$ $=\left(\eta_{1}\left(x, z_{1}\right), \cdots, \eta_{m}\left(x, z_{m}\right)\right)$ is said to be a consistent hair map of $\Omega$, if for any $x \in \partial \Omega$,

(1) $\eta_{i}\left(x, z_{i}\right)$ is a hair map of $\Omega_{i}$ for $i=1, \cdots, m$

(2) $\sum_{i \in I(x)}\left(y_{i} \nabla g_{i}(x)+\eta_{i}\left(x, z_{i}\right)\right)=0, y_{i} \geq 0, z_{i} \geq 0 \quad$ imply $y_{i}=0, z_{i}=0, i \in I(x)$;

(3) $\|(y, z)\| \longrightarrow \infty$ implies $\| \sum_{i \in I(x)}\left(y_{i} \nabla g_{i}(x)+\eta_{i}\left(x, z_{i}\right)\right)$ $\| \longrightarrow \infty$

Definition 8 (pseudocone condition (PCC), see [9]). If there exists a consistent hair map $\eta(x, z)$ of $\Omega$, such that for any $x \in \partial \Omega$,

$$
\left\{x+\sum_{i \in I(x)} \eta_{i}\left(x, z_{i}\right) \mid z_{i} \geq 0, i \in I(x)\right\} \bigcap \Omega^{0}=\varnothing
$$

then, $\Omega$ is said to satisfy the PCC with respect to $\eta(x, z)$.

According to Definition 8, the PCC means that, for any $x \in \partial \Omega$, the set $\left\{x^{0}-x \mid x^{0} \in \Omega^{0}\right\}$ does not intersect with the set $\left\{\sum_{i \in I(x)} \eta_{i}\left(x, z_{i}\right) \mid z_{i} \geq 0, i \in I(x)\right\}$.

In [10], using the following aggregate function introduced in $[11,12]$,

$$
\widehat{g}_{\theta}(x, t)=\theta t \ln \sum_{i=1}^{m} \exp \left(g_{i}(x) / \theta t\right)
$$

where $t>0$ is the smoothing parameter, the parameter $\theta \in(0,1]$, an aggregate constraint homotopy interior point method was proposed. The aggregate constraint homotopy map is defined as 
$H(x, y, t)=\left(\begin{array}{c}(1-t)\left(\nabla f(x)+y \nabla_{x} \widehat{g}_{\theta}(x, t)\right)+t\left(x-x^{0}\right) \\ y \widehat{g}_{\theta}(x, t)-t y^{0} \widehat{g}_{\theta}\left(x^{0}, 1\right)\end{array}\right)$,

where $(x, y, t) \in \Omega \times R_{+} \times(0,1],\left(x^{0}, y^{0}\right) \in \widehat{\Omega} \times R_{++}$, and $\widehat{\Omega}$ is an opened subset of $\Omega^{0}$. Under Assumption 1, the assumption that $\left\{\nabla g_{i}(x) \mid i \in I(x)\right\}$ has a full column rank for any $x \in \partial \Omega$, and the assumption that $\Omega$ satisfies the weak normal cone condition with respect to $\widehat{\Omega}$ (see Definition 9), then, there exists a $\bar{\theta} \in(0,1]$ such that for any $\theta \in(0, \bar{\theta}], \widehat{\Omega} \subset \Omega_{\theta}(t)^{0}=\left\{x \in R^{n} \mid\right.$ $\left.\widehat{g}_{\theta}(x, t)<0\right\} \subset \Omega^{0}$ for $t \in(0,1]$, for almost all $\left(x^{0}, y^{0}\right) \in \widehat{\Omega} \times$ $R_{++}$, the zero point set of (12) defines a smooth homotopy path $\Gamma_{\left(x^{0}, y^{0}\right)}$, which starts from $\left(x^{0}, y^{0}, 1\right)$ and approaches to the hyperplane $t=0$. Moreover, let $\left(x^{*}, y^{*}, 0\right)$ be any limit point of $\Gamma_{\left(x^{0}, y^{0}\right)}$, and $\lambda_{i}^{*}=y^{*} y_{i}\left(x^{*}, 0\right)$ (where $y_{i}\left(x^{*}, 0\right)$ is a limit point of $y_{i}(x, t)=\exp \left(g_{i}(x) / \theta t\right) / \sum_{j=1}^{m} \exp \left(g_{j}(x) /\right.$ $\theta t)$ ) for $i=1, \cdots, m$, then $y^{*}$ is finite, $x^{*}$ is a KKT point of (1), and $\left(\lambda_{1}^{*}, \cdots, \lambda_{m}^{*}\right)$ is the corresponding Lagrangian multiplier.

Definition 9 (weak normal cone condition (WNCC) [10]). If there exists an open subset $\widehat{\Omega} \subset \Omega^{0}$ such that, for any $x \in \partial \Omega$,

$$
\left\{x+\sum_{i \in I(x)} y_{i} \nabla g_{i}(x) \mid y_{i} \geq 0, i \in I(x)\right\} \bigcap \widehat{\Omega}=\varnothing,
$$

then, $\Omega$ is said to satisfy the WNCC with respect to $\widehat{\Omega}$.

According to Definition 9, the WNCC means that there exists an open subset $\widehat{\Omega} \subset \Omega^{0}$ such that for any $x \in \partial \Omega$, the set $\left\{x^{0}-x \mid x^{0} \in \widehat{\Omega}\right\}$ does not intersect with the cone $\left\{\sum_{i \in I(x)} y_{i} \nabla g_{i}(x) \mid y_{i} \geq 0, i \in I(x)\right\}$.

\section{A Comparison of the Four Typical Normal Cone Conditions}

In this section, for the first time, we study the relations of the four typical normal cone conditions introduced in Section 2, and some typical nonconvex sets are introduced.

Proposition 10. If the PLICQ holds, the NCC implies the QNCC and PCC.

Proof. Suppose that the PLICQ holds, then $\eta(x)=\left(\nabla g_{1}(x)\right.$, $\left.\cdots, \nabla g_{m}(x)\right)$ is a positive independent map with respect to $\nabla g(x)$ by Definition 4 , and $\eta(x, z)=\left(z_{1} \nabla g_{1}(x), \cdots, z_{m} \nabla g_{m}\right.$ $(x))$ is a consistent hair map of $\Omega$ by Definition 7. Then, $\Omega$ satisfies the QNCC with respect to $\eta(x)$ by Definition 5, and the PCC with respect to $\eta(x, z)$ by Definition 8 .

Counterexample 3.2. The nonconvex set (see Figure 2) $\Omega=$ $\left\{x \in R^{2} \mid x_{1}^{6}-3 x_{1}^{4}+3 x_{1}^{2}+x_{2}-1 \leq 0,-x_{2} \leq 0\right\}$.

The set $\Omega$ in Counterexample 3.2 satisfies the NCC. However, for $\bar{x}=(1,0) \in \partial \Omega$, we have $I(\bar{x})=\{1,2\}, \nabla g_{1}(\bar{x})$ $=(0,1)$ and $\nabla g_{2}(\bar{x})=(0,-1)$, which means that the PLICQ

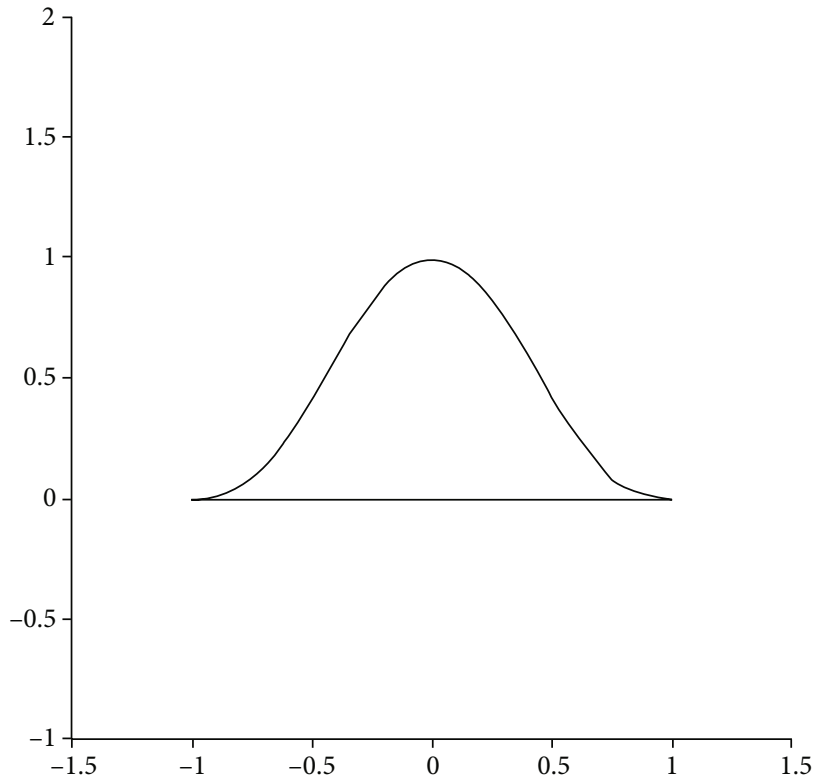

Figure 2: The set in Counterexample 3.2.

does not hold at $(1,0)$. Hence, there does not exist a positive independent map $\eta(x)$ with respect to $\nabla g(x)$ by Definition 4 , and the consistent hair map $\eta(x, z)$ of $\Omega$ by the second item of Definition 7. Therefore, the PLICQ is necessary in Proposition 10.

Proposition 11. The NCC implies the WNCC.

Proof. By Definition 3 and Definition 9, if $\Omega$ satisfies the NCC, $\Omega$ satisfies the WNCC with respect to any open subset $\widehat{\Omega} \subset \Omega^{0}$.

Counterexample 3.4. The nonconvex set (see Figure 3) $\Omega=$ $\left\{x \in R^{2} \mid g_{i}(x) \leq 0, i=1,2,3\right\}$, where

$$
\begin{aligned}
& g_{1}(x)= \begin{cases}4-x_{1}^{2}-x_{2}^{2}, & x_{1}<0, \\
4-x_{2}^{2}, & x_{1} \geq 0,\end{cases} \\
& g_{2}(x)= \begin{cases}x_{1}^{2}+x_{2}^{2}-16, & x_{1}<0, \\
x_{2}^{2}-16, & x_{1} \geq 0,\end{cases} \\
& g_{3}(x)=x_{1}-4 .
\end{aligned}
$$

The set $\Omega$ in Counterexample 3.4 satisfies the QNCC with respect to the positive independent map $\eta(x)=((4-$ $\left.\left.x_{1},-x_{2}\right), \nabla g_{2}(x), \nabla g_{3}(x)\right)$, the PCC with respect to the consistent hair map $\eta(x, z)=\left(z_{1}\left(4-x_{1},-x_{2}\right), z_{2} \nabla g_{2}(x), z_{3} \nabla g_{3}(x)\right)$, and the WNCC with respect to any open subset of $\{x \in \Omega \mid$ $\left.g_{1}(x) \leq 0, g_{2}(x) \leq 0, x_{1} \leq 0\right\}$. For $\bar{x}=(0,2) \in \partial \Omega$ with $I(\bar{x})=$ $\{1\}$ and $\nabla g_{1}(\bar{x})=(0,-4)$, we have $\bar{x}+\bar{y}_{1} \nabla g_{1}(\bar{x})=(0,-3)$ $\in \Omega^{0}$ with $\bar{y}_{1}=5 / 4$, which contradicts (4). Then, we know that the NCC does not hold. Therefore, we have the following result. 


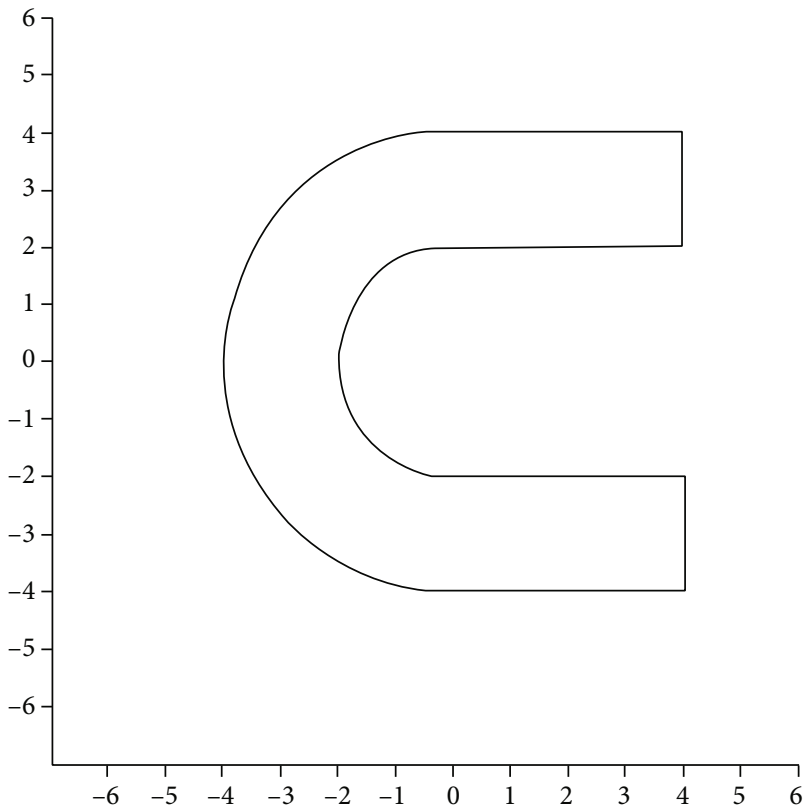

FIgURE 3: The set in Counterexample 3.4.

Remark 12. Any of the QNCC, PCC, and WNCC does not imply the NCC.

Proposition 13. The QNCC implies the PCC.

Proof. If $\Omega$ satisfies the QNCC with respect to the positive independent map $\eta(x)$, we know that $\eta(x, z)=\left(z_{1} \eta_{1}(x), \cdots\right.$, $\left.z_{m} \eta_{m}(x)\right)$ is a consistent hair map of $\Omega$ by Definition 7 . Then, $\Omega$ satisfies the PCC with respect to the consistent hair map $\eta(x, z)$ by Definition 8 .

Counterexample 3.7. The nonconvex set (see Figure 4) $\Omega=$ $\left\{x \in R^{2} \mid g_{i}(x) \leq 0, i=1, \cdots, 10\right\}$, where

$$
\begin{aligned}
& g_{1}(x)=-x_{2}+2, \quad-2 \leq x_{1} \leq 0, x_{2} \geq-5, \\
& g_{2}(x)=-x_{1}-x_{2}+2, \quad 0 \leq x_{1} \leq 1, x_{1}+x_{2} \geq 0, \\
& g_{3}(x)=-x_{2}+1, \quad 1 \leq x_{1} \leq 2, x_{2} \geq-5, \\
& g_{4}(x)=x_{1}-2, \quad 1 \leq x_{2} \leq 3, \\
& g_{5}(x)=x_{2}-3, \\
& g_{6}(x)=-x_{1}-3, \\
& g_{7}(x)=-x_{2}-6, \\
& g_{8}(x)=x_{1}-7.01, \\
& g_{9}(x)=x_{2}+5, \quad x_{1} \geq-2, x_{2} \leq 1, \\
& g_{10}(x)=x_{1}+2, \quad x_{1} \leq 2,-5 \leq x_{2} \leq 2 .
\end{aligned}
$$

For $\bar{x}=(0,2) \in \partial \Omega$ with $I(\bar{x})=\{1,2\}$, and any positive independent map $\eta(x)$, there exists a $\bar{z} \in R_{+}^{10}$ such that

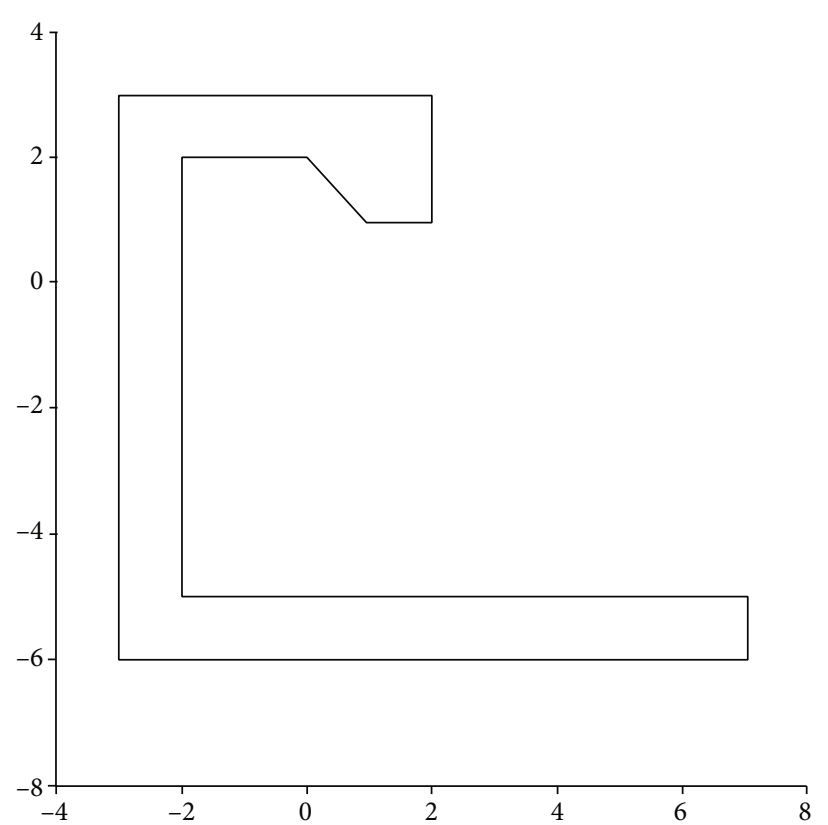

FIgURE 4: The set in Counterexample 3.7.

$\bar{x}+\bar{z}_{1} \eta_{1}(\bar{x})+\bar{z}_{2} \eta_{2}(\bar{x}) \in \Omega^{0}$. Hence, the QNCC does not hold. Define the consistent hair map $\eta(x, z)$ as

$$
\begin{aligned}
& \eta_{1}\left(x, z_{1}\right)=\bar{\eta}_{1}\left(x_{1}, z_{1}\right)-x, x_{1} \in[-2,0], x_{2}=2, \\
& \bar{\eta}_{1}\left(x_{1}, z_{1}\right)= \begin{cases}\left(1-z_{1}\right)\left(x_{1}, 2\right)+z_{1}(1,1), & 0 \leq z_{1} \leq 1, \\
\left(z_{1}, \psi\left(x_{1}, z_{1}\right)\right), & 1<z_{1}<2, \\
\left(z_{1}, 0.5\right), & z_{1} \geq 2,\end{cases}
\end{aligned}
$$

where $\psi(u, v)=((1 / u-1)+1) v^{3}+((5 / u-1)-9 / 2) v^{2}+((8 /$ $u-1)+6) v-(4 / u-1)-3 / 2$ satisfying

$$
\begin{aligned}
\psi(u, 1) & =1, \\
\psi(u, 2) & =0.5, \\
\frac{\partial \psi(u, 1)}{\partial v} & =\frac{1}{u-1}, \\
\frac{\partial \psi(u, 2)}{\partial v} & =0
\end{aligned}
$$

which means that $\bar{\eta}_{1}(u, v)$ is continuously differentiable in $[-2,0] \times[0, \infty)$. For any $u \in[-2,0]$, we have $(1 / u-1)+$ $1 \geq 0, \partial \psi(u, 1) / \partial v=(1 / u-1)<0$ and $\partial \psi(u, 2) / \partial v=0$, and hence

$$
\begin{aligned}
\frac{\partial \psi(u, v)}{\partial v}= & 3\left(\frac{1}{u-1}+1\right) v^{2}-2\left(\frac{5}{u-1}-\frac{9}{2}\right) v+\frac{8}{u-1} \\
& +6<0, v \in(1,2)
\end{aligned}
$$


which means that $\psi(u, v)$ is monotone decreasing for $v \in$ $(1,2)$. By using $\psi(u, v)$, for any $x \in \partial \Omega$ with $x_{1} \in[0,1]$ and $x_{2}=-x_{1}+2$, we define

$$
\begin{aligned}
\eta_{2}\left(x, z_{2}\right) & =\bar{\eta}_{2}\left(x, z_{2}\right)-x, \\
\bar{\eta}_{2}\left(x_{2}, z_{2}\right) & = \begin{cases}\left(1-z_{1}\right) x+z_{2}(1,1), & 0 \leq z_{1} \leq 1, \\
\left(z_{2}, \psi\left(0, z_{2}\right)\right), & 1<z_{1}<2, \\
\left(z_{2}, 0.5\right), & z_{1} \geq 2,\end{cases}
\end{aligned}
$$

then, we know that $\bar{\eta}_{2}(u, v)$ is continuously differentiable in $\left\{(u, v) \in R^{2} \mid u \in[0,1], v=-u+2\right\} \times[0, \infty)$, and for any given $u \in[0,1], \bar{\eta}_{2}(u, v)$ is monotone decreasing for $v \in(0,2)$. For $i=3, \cdots, 10$,

$$
\begin{aligned}
\eta_{3}\left(x, z_{3}\right) & =\left(z_{3}, 0\right), \\
\eta_{i}\left(x, z_{i}\right) & =z_{i} \nabla g_{i}(x), i=4, \cdots, 8, \\
\eta_{9}\left(x, z_{9}\right) & =\left(z_{9}, 0\right), \\
\eta_{10}\left(x, z_{10}\right) & =z_{10}\left(10,-5-x_{2}\right) .
\end{aligned}
$$

For $x \in \partial \Omega$ with $\#(I(x))=1$, we have that (10) holds by the definition of $\eta(x, z)$. For any $x=(2,3),(-3,3),(-3,-6)$, $(7.01,-6) \in \partial \Omega$, we know that (4) holds. Hence, by Proposition 10 and the definitions of $\eta_{i}\left(x, z_{i}\right), i=4, \cdots, 8$, we obtain that (10) also holds.

For $\bar{x}=(0,2) \in \partial \Omega$ with $I(\bar{x})=\{1,2\}$, and any $z_{1} \geq 0$, $z_{2} \geq 0$, we know

$$
\begin{aligned}
\eta_{1}\left(\bar{x}, z_{1}\right)= & \eta_{2}\left(\bar{x}, z_{2}\right) \in\left\{(u, v) \in R^{2} \mid u \in[0,1], v=-u\right\} \bigcup \\
& \cdot\left\{(u, v) \in R^{2} \mid u \in(1, \infty), v \in\left[-\frac{3}{2},-1\right)\right\} .
\end{aligned}
$$

Then, we have

$$
\begin{gathered}
\bar{x}+\eta_{1}\left(\bar{x}, z_{1}\right)+\eta_{2}\left(\bar{x}, z_{2}\right) \in\left\{(u, v) \in R^{2} \mid u \in[0,2], v=-u+2\right\} \bigcup \\
\cdot\left\{(u, v) \in R^{2} \mid u \in(1, \infty), v \in\left[-\frac{1}{2}, 1\right)\right\} \bigcup \\
\cdot\left\{(u, v) \in R^{2} \mid u \in(2, \infty), v \in[-1,0)\right\},
\end{gathered}
$$

and hence, $\bar{x}+\eta_{1}\left(\bar{x}, z_{1}\right)+\eta_{2}\left(\bar{x}, z_{2}\right) \in \Omega^{0}$. For $\bar{x}=(1,1) \in \partial \Omega$ with $I(\bar{x})=\{2,3\}$, and any $z_{2} \geq 0, z_{3} \geq 0$, we know

$\eta_{2}\left(\bar{x}, z_{2}\right) \in\left\{(u, v) \in R^{2} \mid \in[0, \infty), v \in\left[-\frac{1}{2}, 0\right]\right\}, \eta_{3}\left(\bar{x}, z_{3}\right)=\left(z_{3}, 0\right)$.
Then, we have

$\bar{x}+\eta_{2}\left(\bar{x}, z_{2}\right)+\eta_{3}\left(\bar{x}, z_{3}\right) \in\left\{(u, v) \in R^{2} \mid u \in[1, \infty), v \in\left[\frac{1}{2}, 1\right]\right\}$,

and hence, $\bar{x}+\eta_{2}\left(\bar{x}, z_{2}\right)+\eta_{3}\left(\bar{x}, z_{3}\right) \in \Omega^{0}$. For $\bar{x}=(2,1) \in \partial \Omega$ with $I(\bar{x})=\{3,4\}$, and any $z_{3} \geq 0, z_{4} \geq 0$, we know

$$
\eta_{3}\left(\bar{x}, z_{3}\right)=\left(z_{3}, 0\right), \eta_{4}\left(\bar{x}, z_{4}\right)=\left(z_{4}, 0\right) \text {. }
$$

Then, we have

$$
\bar{x}+\eta_{3}\left(\bar{x}, z_{3}\right)+\eta_{4}\left(\bar{x}, z_{4}\right) \in\left\{(u, v) \in R^{2} \mid u \in[2, \infty), v=1\right\},
$$

and hence, $\bar{x}+\eta_{3}\left(\bar{x}, z_{3}\right)+\eta_{4}\left(\bar{x}, z_{4}\right) \in \Omega^{0}$. For $\bar{x}=(7.01,-5)$ $\in \partial \Omega$ with $I(\bar{x})=\{8,9\}$, and any $z_{8} \geq 0, z_{9} \geq 0$, we know

$$
\eta_{8}\left(\bar{x}, z_{8}\right)=\left(z_{8}, 0\right), \eta_{9}\left(\bar{x}, z_{9}\right)=\left(z_{9}, 0\right) \text {. }
$$

Then, we have

$\bar{x}+\eta_{8}\left(\bar{x}, z_{8}\right)+\eta_{9}\left(\bar{x}, z_{9}\right) \in\left\{(u, v) \in R^{2} \mid u \in[7.01, \infty), v=-5\right\}$,

and hence, $\bar{x}+\eta_{8}\left(\bar{x}, z_{8}\right)+\eta_{9}\left(\bar{x}, z_{9}\right) \in \Omega^{0}$. For $\bar{x}=(-2,-5) \epsilon$ $\partial \Omega$ with $I(\bar{x})=\{9,10\}$, and any $z_{8} \geq 0, z_{9} \geq 0$, we know

$$
\eta_{9}\left(\bar{x}, z_{9}\right)=\left(z_{9}, 0\right), \eta_{10}\left(\bar{x}, z_{10}\right)=\left(10 z_{10}, 0\right) .
$$

Then, we have

$$
\bar{x}+\eta_{9}\left(\bar{x}, z_{9}\right)+\eta_{10}\left(\bar{x}, z_{10}\right) \in\left\{(u, v) \in R^{2} \mid u \in[-2, \infty), v=-5\right\},
$$

and hence, $\bar{x}+\eta_{9}\left(\bar{x}, z_{9}\right)+\eta_{10}\left(\bar{x}, z_{10}\right) \in \Omega^{0}$. For $\bar{x}=(-2,2) \epsilon$ $\partial \Omega$ with $I(\bar{x})=\{1,10\}$, and any $z_{1} \geq 0, z_{10} \geq 0$, we know

$$
\begin{gathered}
\eta_{1}\left(\bar{x}, z_{1}\right) \in\left\{(u, v) \in R^{2} \mid u \in[0,3], v=-\frac{1}{3} u\right\} \bigcup \\
\cdot\left\{(u, v) \in R^{2} \mid u \in(3, \infty), v \in\left[-\frac{3}{2},-1\right)\right\}, \\
\eta_{10}\left(\bar{x}, z_{10}\right)=z_{10}(10,-7) .
\end{gathered}
$$

Then, we have

$$
\begin{aligned}
\bar{x}+ & \eta_{1}\left(\bar{x}, z_{1}\right)+\eta_{10}\left(\bar{x}, z_{10}\right) \in \\
\cdot & \left\{(u, v) \in R^{2} \mid u \in[-2, \infty),-\frac{7}{10} u+\frac{3}{5} \leq v \leq-\frac{1}{3} u+\frac{4}{3}\right\} \bigcup \\
\cdot & \left\{(u, v) \in R^{2} \mid u \in(1, \infty),-\frac{7}{10} u+\frac{6}{5}<v<1\right\},
\end{aligned}
$$


and hence, $\bar{x}+\eta_{1}\left(\bar{x}, z_{1}\right)+\eta_{10}\left(\bar{x}, z_{10}\right) \in \Omega^{0}$. Therefore, the PCC holds with respect to the consistent hair map $\eta(x, z)$, and hence we have the following result.

Remark 14. The PCC does not imply the QNCC.

Counterexample 3.9. The nonconvex set (see Figure 5) $\Omega=$ $\left\{x \in R^{2} \mid g_{i}(x) \leq 0, i=1, \cdots, 5\right\}$, where

$$
\begin{aligned}
& g_{1}(x)=-x_{1}-6, \\
& g_{2}(x)=x_{1}-6, \\
& g_{3}(x)=-x_{2}-10, \\
& g_{4}(x)=x_{2}-10, \\
& g_{5}(x)= \begin{cases}-\frac{x_{1}^{2}}{25}-\frac{x_{2}^{2}}{81}+1, & x_{1}<\frac{5 \sqrt{2}}{2}, \\
\left(\sqrt{2}-\frac{1}{5} x_{1}\right)^{2}-\frac{x_{2}^{2}}{81}, & x_{1} \geq \frac{5 \sqrt{2}}{2} .\end{cases}
\end{aligned}
$$

The set $\Omega$ in Counterexample 3.9 satisfies the QNCC with respect to the positive independent map $\eta(x)=\left(\nabla g_{1}\right.$ $\left.(x), \cdots, \nabla g_{4}(x),\left(6-x_{1},-x_{2}\right)\right)$, and the PCC with respect to the hair map $\eta(x, z)=\left(z_{1} \nabla g_{1}(x), \cdots, z_{4} \nabla g_{4}(x), z_{5}\left(6-x_{1}\right.\right.$,$\left.x_{2}\right)$. For any $x^{0} \in \Omega^{0}$, let $k_{1}(x)=\left(\partial g_{5}(x) / \partial x_{2}\right) /\left(\partial g_{5}(x) / \partial x_{1}\right)$ for $x \in\left\{x \in \partial \Omega \mid\left(\partial g_{5}(x) / \partial x_{1}\right) \neq 0\right\}, \quad k_{2}(x)=x_{2}-x_{2}^{0} / x_{1}-x_{1}^{0}$ for $x \in\left\{x \in \partial \Omega \mid x_{1}-x_{1}^{0} \neq 0\right\}$. For convenience, we rewrite $\Omega^{0}$ as $\Omega^{0}=\bar{\Omega}_{1} \bigcup \bar{\Omega}_{2} \bigcup \bar{\Omega}_{3} \bigcup \bar{\Omega}_{4} \bigcup \bar{\Omega}_{5} \bigcup \bar{\Omega}_{6}$ with $\bar{\Omega}_{1}=\{(u$, $\left.v) \in \Omega^{0} \mid u>0, v>0\right\}, \bar{\Omega}_{2}=\left\{(0, v) \in \Omega^{0} \mid v>0\right\}, \bar{\Omega}_{3}=\{(u, v)$ $\left.\in \Omega^{0} \mid u<0, v \geq 0\right\}, \quad \bar{\Omega}_{4}=\left\{(u, v) \in \Omega^{0} \mid u<0, v \leq 0\right\}, \quad \bar{\Omega}_{5}=$ $\left\{(0, v) \in \Omega^{0} \mid v<0\right\}, \quad \bar{\Omega}_{6}=\left\{(u, v) \in \Omega^{0} \mid u>0, v<0\right\}$.

For any $x^{0} \in \bar{\Omega}_{2}, \bar{y}_{5}=9\left(x_{2}^{0}+9\right) / 2>0$, and $\bar{x}=(0,-9) \in \partial \Omega$ with $I(\bar{x})=\{5\}$, we have

$$
\bar{x}+\bar{y}_{5} \nabla g_{5}(\bar{x})=(0,-9)+\frac{9\left(x_{2}^{0}+9\right)}{2}\left(0, \frac{2}{9}\right)=x^{0} .
$$

For any $x^{0} \in \bar{\Omega}_{5}$, let $\bar{y}_{5}=9\left(9-x_{2}^{0}\right) / 2>0, \bar{x}=(0,9) \in \partial \Omega$ with $I(\bar{x})=\{5\}$, we have

$$
\bar{x}+\bar{y}_{5} \nabla g_{5}(\bar{x})=(0,9)+\frac{9\left(9-x_{2}^{0}\right)}{2}\left(0,-\frac{2}{9}\right)=x^{0}
$$

For any $x^{0} \in \bar{\Omega}_{3}$ and $x \in\left\{x \in \partial \Omega \mid g_{5}(x)=0,0<x_{1} \leq 5\right.$ $\left.\sqrt{2} / 2, x_{2} \leq-9 \sqrt{2} / 2\right\}, k_{1}(x)$ and $k_{2}(x)$ reduce to $\bar{k}_{1}\left(x_{1}\right)=-5$ $\sqrt{25-x_{1}^{2}} / 9 x_{1}$ and $\bar{k}_{2}\left(x_{1}\right)=-9 \sqrt{25-x_{1}^{2}}-5 x_{2}^{0} / 5\left(x_{1}-x_{1}^{0}\right)$, respectively. By the continuity of $\bar{k}_{1}\left(x_{1}\right)$ and $\bar{k}_{2}\left(x_{1}\right)$ on $(0,5$ $\sqrt{2} / 2), \quad \lim _{x_{1} \rightarrow 0^{+}}\left(\bar{k}_{1}\left(x_{1}\right)-\bar{k}_{2}\left(x_{1}\right)\right) \rightarrow-\infty<0, \quad \bar{k}_{1}(5 \sqrt{2} / 2)-\bar{k}_{2}(5$ $\sqrt{2} / 2)>0$ coming from $\bar{k}_{1}(5 \sqrt{2} / 2)=-5 / 9$ and $\bar{k}_{2}(5 \sqrt{2} / 2)$

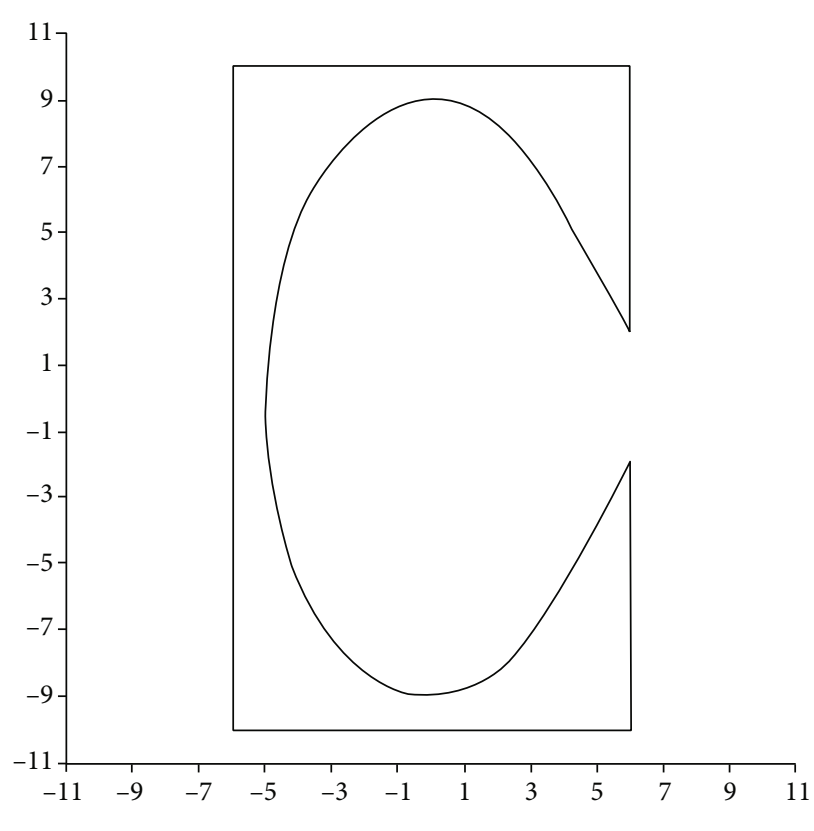

FIgURe 5: The set in Counterexample 3.9.

$\epsilon(-9 / 5-2 \sqrt{2},-9 / 47(6 \sqrt{2}-5)]$, there exists an $\bar{x}_{1} \in(0,5$ $\sqrt{2} / 2)$ such that $k_{1}\left(\bar{x}_{1}\right)=k_{2}\left(\bar{x}_{1}\right)$, which means

$$
\frac{25\left(\bar{x}_{1}-x_{1}^{0}\right)}{2 \bar{x}_{1}}=\frac{81 \sqrt{25-\bar{x}_{1}^{2}}+45 x_{2}^{0}}{2 \sqrt{25-\bar{x}_{1}{ }^{2}}} .
$$

Then, for $\bar{x}=\left(\bar{x}_{1},-9 \sqrt{25-\bar{x}_{1}^{2}} / 5\right) \in \partial \Omega$ with $I(\bar{x})=\{5\}$, $\bar{y}_{5}=25\left(\bar{x}_{1}-x_{1}^{0}\right) / 2 \bar{x}_{1}=\left(81 \sqrt{25-\bar{x}_{1}^{2}}+45 x_{2}^{0}\right) /\left(2 \sqrt{25-\bar{x}_{1}^{2}}\right)$

$>0$, we have

$$
\begin{aligned}
\bar{x}+\bar{y}_{5} \nabla g_{5}(\bar{x})= & \left(\bar{x}_{1},-\frac{9 \sqrt{25-\bar{x}_{1}^{2}}}{5}\right)+\bar{y}_{5}\left(-\frac{2 \bar{x}_{1}}{25}, \frac{2 \sqrt{25-\bar{x}_{1}^{2}}}{45}\right) \\
= & \left(\bar{x}_{1}-\frac{25\left(\bar{x}_{1}-x_{1}^{0}\right)}{2 \bar{x}_{1}} \frac{2 \bar{x}_{1}}{25},-\frac{9 \sqrt{25-\bar{x}_{1}^{2}}}{5}\right. \\
& \left.+\frac{81 \sqrt{25-\bar{x}_{1}^{2}}+45 x_{2}^{0}}{2 \sqrt{25-\bar{x}_{1}^{2}}} \frac{2 \sqrt{25-\bar{x}_{1}^{2}}}{45}\right) \\
= & \left(x_{1}^{0},-\frac{9 \sqrt{25-\bar{x}_{1}^{2}}}{5}+\frac{9 \sqrt{25-\bar{x}_{1}^{2}}+5 x_{2}^{0}}{5}\right)=x^{0}
\end{aligned}
$$

Analogously, for any $x^{0} \in \bar{\Omega}_{1} \subset\left\{x \in R^{2} \mid\left(-x_{1}, x_{2}\right) \in \bar{\Omega}_{3}\right\}$, $\bar{\Omega}_{4}$ and $\bar{\Omega}_{6} \subset\left\{x \in R^{2} \mid-x \in \bar{\Omega}_{3}\right\}$, there exist an $\bar{x} \in \partial \Omega$ with $I(\bar{x})=\{5\}$ and a $\bar{y}_{5}>0$ such that

$$
\bar{x}+\bar{y}_{5} \nabla g_{5}(\bar{x})=x^{0}
$$

Therefore, the WNCC does not hold, and hence we have the following result.

Remark 15. The QNCC or PCC does not imply the WNCC. 


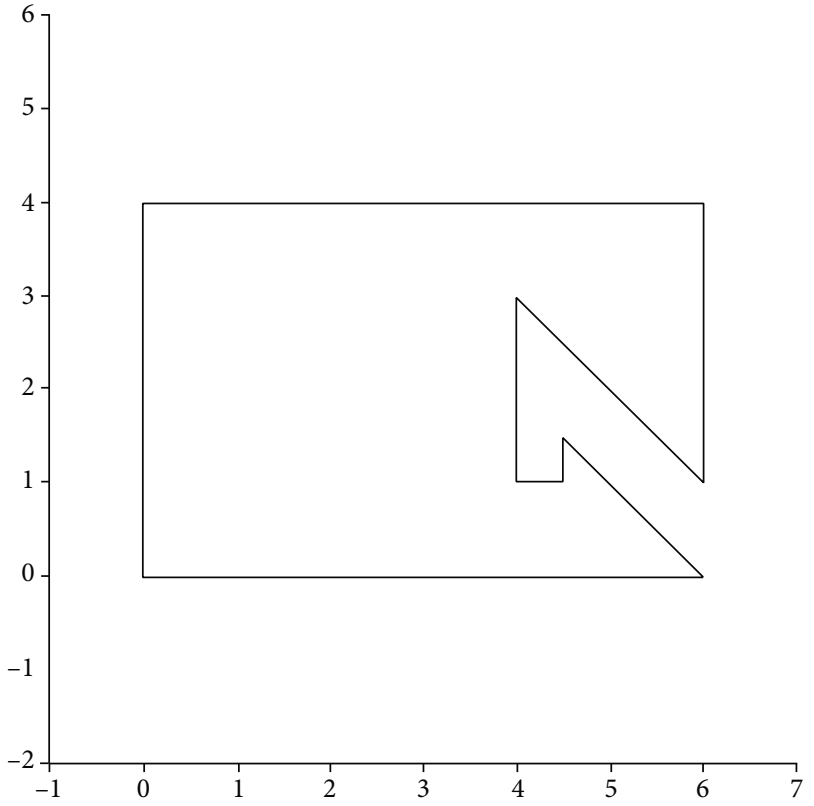

FIgURE 6: The set in Counterexample 3.11.

Counterexample 3.11. The nonconvex set (see Figure 6) $\Omega=$ $\left\{x \in R^{2} \mid g_{i}(x) \leq 0, i=1, \cdots, 9\right\}$, where

$$
\begin{aligned}
& g_{1}(x)=-x_{1}, \\
& g_{2}(x)=x_{1}-6, \\
& g_{3}(x)=-x_{2}, \\
& g_{4}(x)=x_{2}-4, \\
& g_{5}(x)=-x_{1}-x_{2}+7, \quad 4 \leq x_{1} \leq 6, x_{1}+x_{2} \geq 6, \\
& g_{6}(x)=x_{1}+x_{2}-6, \quad x_{1}+x_{2} \leq 7,4.5 \leq x_{2} \leq 6, \\
& g_{7}(x)=x_{1}-4, \quad x_{1} \leq 4,1 \leq x_{2} \leq 3, \\
& g_{8}(x)=-x_{1}+4.5, \quad x_{1} \geq 4,1 \leq x_{2} \leq 1.5, \\
& g_{9}(x)=x_{2}-1, \quad 4 \leq x_{1} \leq 4.5, x_{2} \leq 2 .
\end{aligned}
$$

The set $\Omega$ in Counterexample 3.11 satisfies the WNCC with respect to any open subset of $\left\{x \in R^{2} \mid x_{1}>0,0<x_{2}<1\right.$, $\left.x_{1}-x_{2}-1<0\right\} \subset \Omega^{0}$. For $\bar{x}=(4.5,1.4) \in \partial \Omega$ with $I(\bar{x})=$ $\{8\}$ and $\nabla g_{8}(\bar{x})=(-1,0)$, and any hair map $\eta(x, z)$ satisfying (10), we have $\bar{x}+\eta_{8}(\bar{x}, 0)=\bar{x}$ and $\lim _{z_{8} \rightarrow \infty}\left\|\bar{x}+\eta_{8}\left(\bar{x}, z_{8}\right)\right\|=$ $\infty$ by Definition 6 . Hence, there exists a $\bar{z}_{8}>0$ such that $\bar{x}+\eta_{8}\left(\bar{x}, \bar{z}_{8}\right) \in\left\{x \in R^{2} \mid 4.6 \leq x_{1} \leq 5.6, x_{2}=1.4\right\}$. Then, we know $\eta_{8}\left(\bar{x}, \bar{z}_{8}\right) \in\left\{x \in R^{2} \mid 0.1 \leq x_{1} \leq 1.1, x_{2}=0\right\}$ and $\bar{y}_{8} \nabla g_{8}$ $(\bar{x})+\eta_{8}\left(\bar{x}, \bar{z}_{8}\right)=0$ with $\bar{y}_{8}=\left(\eta_{8}\left(\bar{x}, \bar{z}_{8}\right)\right)_{1}>0$, which contradicts the second item of Definition 7 . Therefore, there does not exist a consistent hair map $\eta(x, z)$ with respect to $\Omega$ to satisfy the PCC, and the QNCC also does not hold by Proposition 13. Then, we obtain the following result.

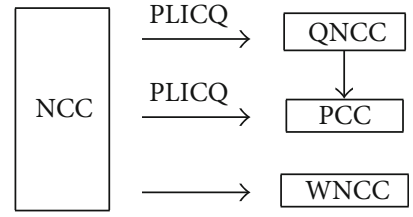

FIGURE 7: Relations among the four typical normal cone conditions.

Remark 16. The WNCC does not imply the QNCC or PCC.

In conclusion, we show the relations among the four typical normal cone conditions in Figure 7. It is noted that there still exist many nonconvex sets satisfying none of existing normal cone conditions, such as the nonconvex set in Counterexample 3.13.

Counterexample 3.13. The nonconvex set (see Figure 8) $\Omega=\left\{x \in R^{2} \mid-x_{1}^{2}-x_{2}^{2}+2.25 \leq 0, x_{1}^{2}+x_{2}^{2}-9 \leq 0\right\}$.

\section{The Global Convergence of the Homotopy Methods for Solving ICNLP}

As shown in Section 2, the global convergence of these homotopy methods can be established under three conditions. In this section, we present some comments for these conditions.

The boundedness of $\Omega^{0}$ ensures that the variable $x$ in the homotopy path keeps bounded. For Example $4.1, \Omega^{0}=$ $\left\{x \in R^{2} \mid x_{1}^{2}+x_{2}<0\right\}$ is unbounded, we have $x \longrightarrow(-\infty,-$ $\infty)$ with $y \longrightarrow 0$ and $t \rightarrow 0$ for the starting point $x^{0}=(0,-1)$ with $y^{0}=1$ in the homotopy path defined by the CHIP method. Since the real-world ICNLP problems generally have the optimal solutions at finity, the variable $x$ in the homotopy path always keeps bounded even if $\Omega^{0}$ is unbounded. For Example 4.2, $\Omega^{0}=\left\{x \in R^{2} \mid x_{1}^{2}-x_{2}<0\right\}$ is unbounded, but we have $x \longrightarrow(-0.5,0.25)$ with $y \longrightarrow 1$ and $t \longrightarrow 0$ for the starting point $x^{0}=(0,1)$ with $y^{0}=1$ in the homotopy path defined by the CHIP method.

Example 4.1.

$$
\begin{array}{ll}
\min _{x \in R^{2}} & f(x)=x_{1}+x_{2}, \\
\text { s.t. } & g(x)=x_{1}^{2}+x_{2} \leq 0 .
\end{array}
$$

Example 4.2.

$$
\begin{array}{ll}
\min _{x \in R^{2}} & f(x)=x_{1}+x_{2}, \\
\text { s.t. } & g(x)=x_{1}^{2}-x_{2} \leq 0 .
\end{array}
$$

The PLICQ (MFCQ) is the most widely used constraint qualification for the ICNLP problems, it ensures that the variable $y$ in the homotopy path keeps bounded. For Example 4.3, the PLICQ does not hold at $(0,-1) \in \partial \Omega$, we have $x \longrightarrow(0,-1)$ that is not a KKT point, $y \longrightarrow(0,+\infty,+$ $\infty)$ and $t \longrightarrow 0$ for the starting point $x^{0}=(-2,0)$ with $y^{0}=$ $(1,1,1)$, and $x \longrightarrow(2.8242,-1), y \longrightarrow(0.1768,0,0.6464)$ 


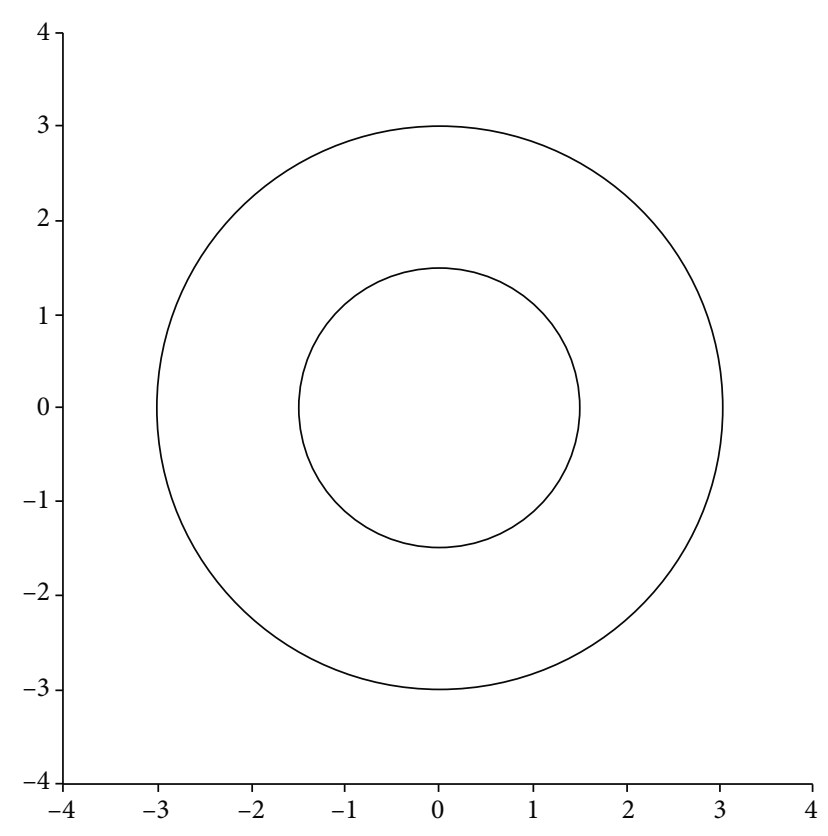

Figure 8: The set in Counterexample 3.13.

and $t \longrightarrow 0$ for the starting point $x^{0}=(2,0)$ with $y^{0}=(1,1,1)$ in the homotopy path defined by the CHIP method.

Example 4.3.

$$
\begin{array}{ll}
\min _{x \in R^{2}} & f(x)=-x_{1}+x_{2}, \\
& g_{1}(x)=x_{1}^{2}+x_{2}^{2}-9 \leq 0, \\
\text { s.t. } & g_{2}(x)=-x_{1}^{2}-x_{2}^{2}+1 \leq 0, \\
& g_{3}(x)=-x_{2}-1 \leq 0 .
\end{array}
$$

The normal cone conditions and the PLICQ ensure that the variable $y$ in the homotopy path keeps bounded when the homotopy path tends to the hyperplane $\{(x, y, 1) \mid(x, y)$ $\left.\in \Omega \times R_{+}^{m}\right\}$. Although our numerical experiences show that these homotopy methods always globally converge even if the normal cone conditions do not hold, the normal cone conditions are important to establish the global convergence of these homotopy methods in theory. For Example 4.4, the normal cone conditions do not hold for any $x \in\left\{x \in R^{2}\right.$ $\left.x_{1}^{2}+x_{2}^{2}=1\right\} \subset \partial \Omega$, but we have $x \longrightarrow(2,-2), y \longrightarrow(0.25,0)$ and $t \longrightarrow 0$ for all tested starting points $x^{0} \in \Omega^{0}$ with $y^{0}=$ $(1,1)$ in the homotopy path defined by the CHIP method.

Example 4.4 .

$$
\begin{array}{ll}
\min _{x \in R^{2}} & f(x)=-x_{1}+x_{2}, \\
\text { s.t. } & g_{1}(x)=x_{1}^{2}+x_{2}^{2}-8 \leq 0, \\
& g_{2}(x)=-x_{1}^{2}-x_{2}^{2}+1 \leq 0 .
\end{array}
$$

\section{Conclusion}

In this paper, we provide a comparison of four typical normal cone conditions used in homotopy methods for solving inequality constrained nonlinear programming. The NCC holds for convex sets and a class of nonconvex sets. The WNCC, QNCC, and PCC are more weak than the NCC. However, the NCC is more convenient to use than the WNCC, QNCC and PCC. For the feasible set satisfying the QNCC or PCC, some auxiliary maps should be constructed in the homotopy maps. For the feasible set satisfying the WNCC, the starting point for the homotopy methods should be chosen from a special open subset of the feasible set. On the other hand, except for few special nonconvex sets, there does not exist a general way of checking a set to satisfy the NCC or construct the open subset, the positive independent map and the consistent hair map for the WNCC, QNCC, and PCC, respectively.

\section{Data Availability}

The data used to support the findings of this study are available from the corresponding author upon request.

\section{Conflicts of Interest}

The authors declare that there is no conflict of interest regarding the publication of this paper.

\section{Acknowledgments}

This study was supported by the National Natural Science Foundation of China (No. 11701350).

\section{References}

[1] E. L. Allgower and K. Georg, "Numerical path following," in Handbook of Numerical Analysis, Vol. 5, P. G. Ciarlet and J. L. Lions, Eds., North Holland Publishing Company, 1997.

[2] E. L. Allgower and K. Georg, "Introduction to Numerical Continuation Methods," in Classics in Applied Mathematics, Vol. 45, Society for Industrial and Applied Mathematics, Philadelphia, PA, USA, 2003.

[3] C. B. Garcia and W. I. Zangwill, Pathways to Solutions, Fixed Points and Equilibria, Prentice-Hall, Englewood Cliffs, NJ, USA, 1981.

[4] G. C. Feng and B. Yu, "Combined homotopy interior point method for nonlinear programming problems," Advances in Numerical Mathematics, pp. 9-16, 1995.

[5] G. C. Feng, Z. H. Lin, and B. Yu, "Existence of an interior pathway to a Karush-Kuhn-Tucker point of a nonconvex programming problem," Nonlinear Analysis: Theory, Methods \& Applications, vol. 32, no. 6, pp. 761-768, 1998.

[6] N. H. Chieu, V. Jeyakumar, G. Li, and H. Mohebi, "Constraint qualifications for convex optimization without convexity of constraints : new connections and applications to best approximation," European Journal of Operational Research, vol. 265, no. 1, pp. 19-25, 2018.

[7] L. T. Watson, "Theory of globally convergent probability-one homotopies for non-linear programming," SIAM Journal on Optimization, vol. 11, no. 3, pp. 761-780, 2001. 
[8] Q. H. Liu, B. Yu, and G. C. Feng, "An interior point pathfollowing method for nonconvex programming with quasi normal cone condition," Advances in Mathematics, vol. 29, no. 4, pp. 281-282, 2000.

[9] B. Yu, Q. H. Liu, and G. C. Feng, "A combined homotopy interior point method for nonconvex programming with pseudo cone condition," Northeastern Mathematical Journal, vol. 16, no. 4, pp. 383-386, 2000.

[10] B. Yu, G. C. Feng, and S. L. Zhang, "The aggregate constraint homotopy method for nonconvex nonlinear programming," Nonlinear Analysis: Theory, Methods \& Applications, vol. 45, no. 7, pp. 839-847, 2001.

[11] X. S. Li, "An aggregate function method for nonlinear programming," Science in China Series A, vol. 34, no. 12, pp. 1467-1473, 1991.

[12] X. S. Li, “An aggregate constraint method for nonlinear programming," The Journal of the Operational Research Society, vol. 42, no. 11, pp. 1003-1010, 2017. 